\title{
Occlusal Stress Distribution on the Mandibular First Premolar - FEM Analysis
}

\author{
Amra Ahmić Vuković ${ }^{1}$, Selma Jakupović2, Selma Zukić1, Anita Bajsman ${ }^{1}$, \\ Alma Gavranović Glamoč ${ }^{3}$, Sadeta Šečić ${ }^{4}$
}

${ }^{1}$ Department of Dental Morphology with Dental Anthropology and Forensics, Faculty of Dentistry, University of Sarajevo, ${ }^{2}$ Department of Restorative Dentistry and Endodontics, Faculty of Dentistry, University of Sarajevo, ${ }^{3}$ Department of Prostodontics, Faculty of Dentistry, University of Sarajevo, ${ }^{4}$ Department of Oral Surgery, Faculty of Dentistry, University of Sarajevo

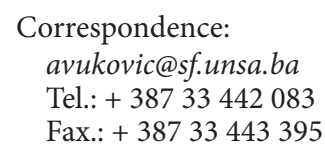

Received: 26 March 2019; Accepted: 17 November 2019

\section{Abstract}

Objective. The aim of this paper was to analyze the distribution of stress and deformation on the mandibular first premolar under two types of loading (axial and para-axial load of $200 \mathrm{~N}$ ) using the FEM computer method. Materials and Method. For this research a $\mu \mathrm{CT}$ scan of the first mandibular premolar was used, and the method used in this research was FEM analysis under two types of loading. Results. The values of the von Mises stress measured in the cervical part of an intact tooth under axial load were up to 12 $\mathrm{MPa}$, and under paraaxial load over $50 \mathrm{MPa}$. The values of the stress measured on the bottom of the noncarious lesion are very high $\approx 240 \mathrm{Mpa}$. Stress values in the cervical part of the intact tooth are higher in the zone of the sub-surface enamel. The deformation values of the tooth under para-axial loading were $\approx 10$ times higher than the value of the deformation under axial load. The greatest deformations were seen in the area of the tooth crown. Conclusions. Occlusal loading leads to significant stress in the cervical part of
\end{abstract}

teeth. The values of the measured stress are greater under the action of paraxial load. The values of stress in abfraction lesions measured under a paraxial load are extremely high. Exposing the lesion to further stress will lead to its deepening. The total deformation of the entire tooth under paraxial load was $\approx 10$ times higher compared to the deformation value of the tooth under axial load.

Key Words: Mandibular first premolar - Morphology - Abfraction - FEM - Stress - Deformation.

\section{Introduction}

\section{Morphological Characteristics of the Mandibular First Premolar}

The mandibular first premolar is a specific tooth in form and function. It could be considered as a transitional form between the lower canine and the mandibular second premolar because it has some characteristics of both. Although it has the morphological characteristics of the posterior teeth, the lower first premolar has a masticatory function similar to the canine (1). Due to the lingual inclination of its crown, its occlusal surface does not lie perpendicular to the long axis of the root (2). The mandibular first premolar has a large buccal cusp with the tip located near the center of the occlusal surface, and it is only an occluding cusp. The contact of the buccal cusp during articula- 
tion and/or occlusion with an antagonist tooth is not on its very tip, but rather on the buccal surface of the buccal cusp. Having in mind the prominent crown inclination of the first mandibular premolar and its specific contact area, it is easy to pose the question on their contribution to the mechanism of the distribution of occlusal forces. The durability of teeth depends on occlusal forces and the distribution of those loads.

The mandibular first premolar is the tooth with the highest prevalence of noncarious cervical lesions, as determined in previous studies. (3-6). A specific clinical phenomenon called abfraction is related to the distribution of stress along the tooth. An abfraction lesion is a type of noncarious cervical lesion (NCCL) in the form of the microstructural loss of dental tissue, due to the action of biomechanical occlusal forces in the area of the highest stress concentration - the cervical region. This loss of hard dental tissue in the cervical part of the tooth was termed abfraction by Grippo (7) in 1991, precisely to make a distinct from lesions caused by erosion and abrasion. The incidence and severity of noncarious cervical lesions increase with the age of the patient $(8,3)$. The presence of noncarious cervical lesions of different morphologies influences the biomechanics of the cervical tooth region. A three-dimensional finite element analysis (FEM) proved to be a good experimental model in research to help understand the complex biomechanics of different tooth regions in other teeth (9). The aim of this paper was to evaluate the influence of different occlusal loads on the mandibular first premolar in the process of the formation of cervical lesions. FEM analysis enables an understanding of the complex process of development and progression of NCCL, considering the specific morphology of mandibular first premolars.

\section{Material and Methods}

A mandibular first premolar was scanned by micro computed tomography $(\mu \mathrm{CT})$ scanner (SkyScan 1076 Kontich, Belgium). A volumetric 3D CAD tooth model was created using program packages MATLAB (MathWorks, Inc., Natick, USA) and Creo Parametric 1.0 CAD software. On one tooth model a wedge-shaped abfraction lesion, a- V lesion, was modeled. The models were exported to the finite element analysis software ANSYS Workbench (14.0) and a finite element mesh of the models was made by dividing the tooth into a large, but finite, number of smaller structural triangle elements, connected by nodal points. The properties of the tooth material, Young's modulus and Poisson's ratio were used for all tooth tissues. The model was fixed to allow displacement under load by $300 \mu \mathrm{m}$, equal to the average thickness of the periodontal ligament. The presence of contact points with adjacent teeth was also simulated. Axial and para-axial loads of $200 \mathrm{~N}$ were simulated on the models. To record the complex stress measured in (MPa), von Mises stress (VMS) was used.

A detailed description of the methodology of the study was given in our paper published in Eur J Dent 2016; 10: 413-8. Biomechanics of the cervical tooth region and noncarious cervical lesions of different morphologies; a three-dimensional finite element analysis / Published by Wolters Kluwer - Medknow (9).

\section{Results}

Stress distribution, as well as the type of deformation, differs in intact first mandibular premolars compared to those with noncarious cervical lesions, and depends on the type of loading (axial or paraxial). A paraxial load of $200 \mathrm{~N}$ causes almost five times higher stress in the cervical part of an intact 


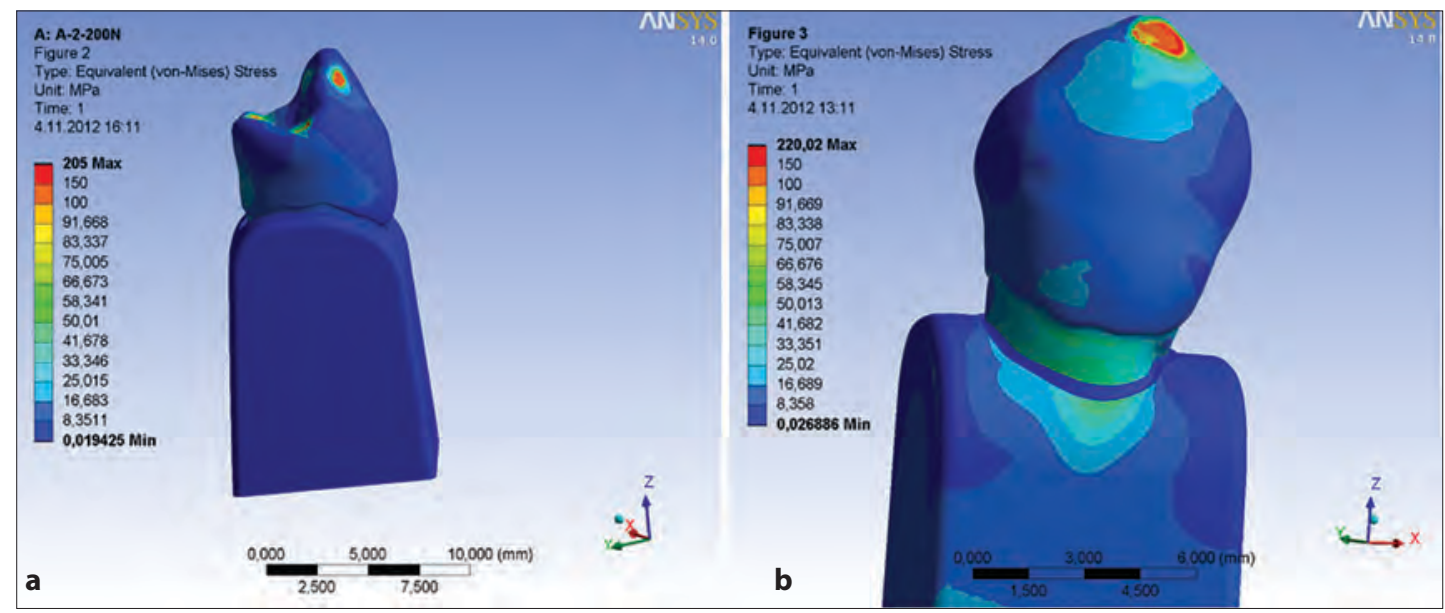

Figure 1. Stress distribution in a mandibular first premolar under axial (a) and paraxial loads (b) of $200 \mathrm{~N}$. The values of von Mises stress measured in the cervical part of the intact tooth under axial load rise up to $12 \mathrm{MPa}$, while the stress in the same area of the tooth is almost 5 times higher under the action of paraxial forces, and is over $50 \mathrm{MPa}$.

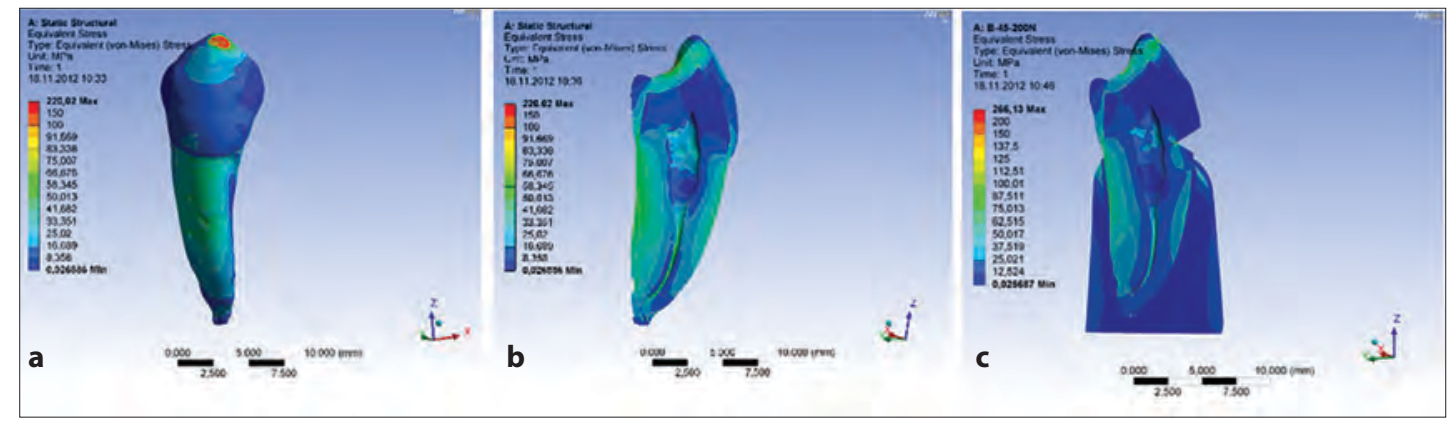

Figure 2. Stress distribution in a mandibular first premolar without lesions ( $a$ and $b$ ), and with an existing lesion (c) under a $200 \mathrm{~N}$ paraxial load. The values of the stress measured inside the tooth model with an abfraction lesion are higher within all dental tissues compared to the intact tooth.

first mandibular premolar compared to an axial load of the same intensity (Figure 1). On a tooth with a cervical lesion, the stress caused by a paraxial load inside all dental tissues becomes even higher (Figure 2). The stress measured on the non-carious cervical lesion is the highest at its bottom $(\approx 240$ $\mathrm{MPa})$. Although significantly lower stress is presented in an intact tooth, it becomes higher in the zone of the sub-surface cervical enamel, suggesting that the initial fracture of the enamel could occur in these layers (Figure 3).
A paraxial load also causes tensile stress (+) on the buccal side of the intact tooth and compressive stress (-) on the oral side (Figure 4). The values of the deformation of the tooth under para-axial loading were $\approx$ 10 times higher than the deformation of the tooth under axial load. The greatest deformations were seen in the area of the tooth crown (Figure 5). Different types of loading cause different types of deformation in a tooth with a cervical lesion. When paraxial load occurs on the tooth with a cervical lesion the deformation is greater $(0.176 \mathrm{~mm})$ 


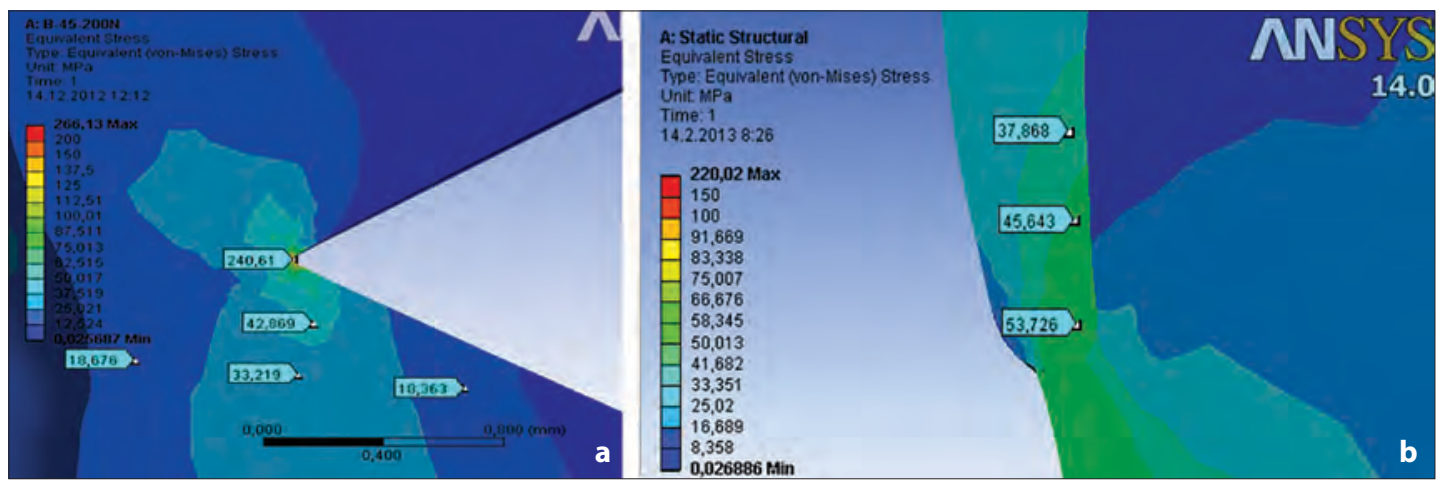

Figure 3. Stress distribution on the sagittal section of a tooth with a lesion (a) and a tooth without a lesion (b) under a paraxial load of $200 \mathrm{~N}$. The values of the stress measured at the bottom of the non-carious lesion are very high ( $\approx 240 \mathrm{MPa}$ ). It was noted that the stress values in the cervical part of the intact tooth are higher in the zone of the sub-surface enamel, suggesting that the initial fracture of the enamel could occur in these layers.

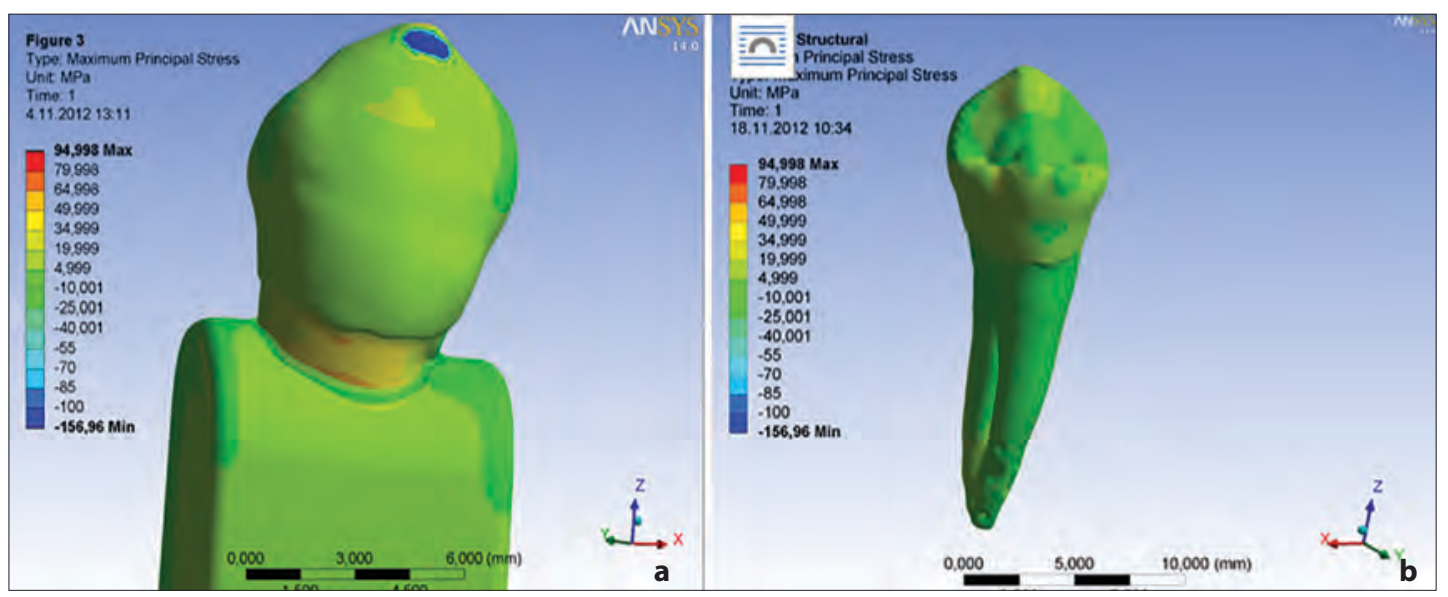

Figure 4. Distribution of principal stresses (Maximum Principal Stress) on the intact tooth. Under paraxial load, tensile stress (+) was present on the buccal side of the tooth (a) and compressive stress (-) on the oral side (b).

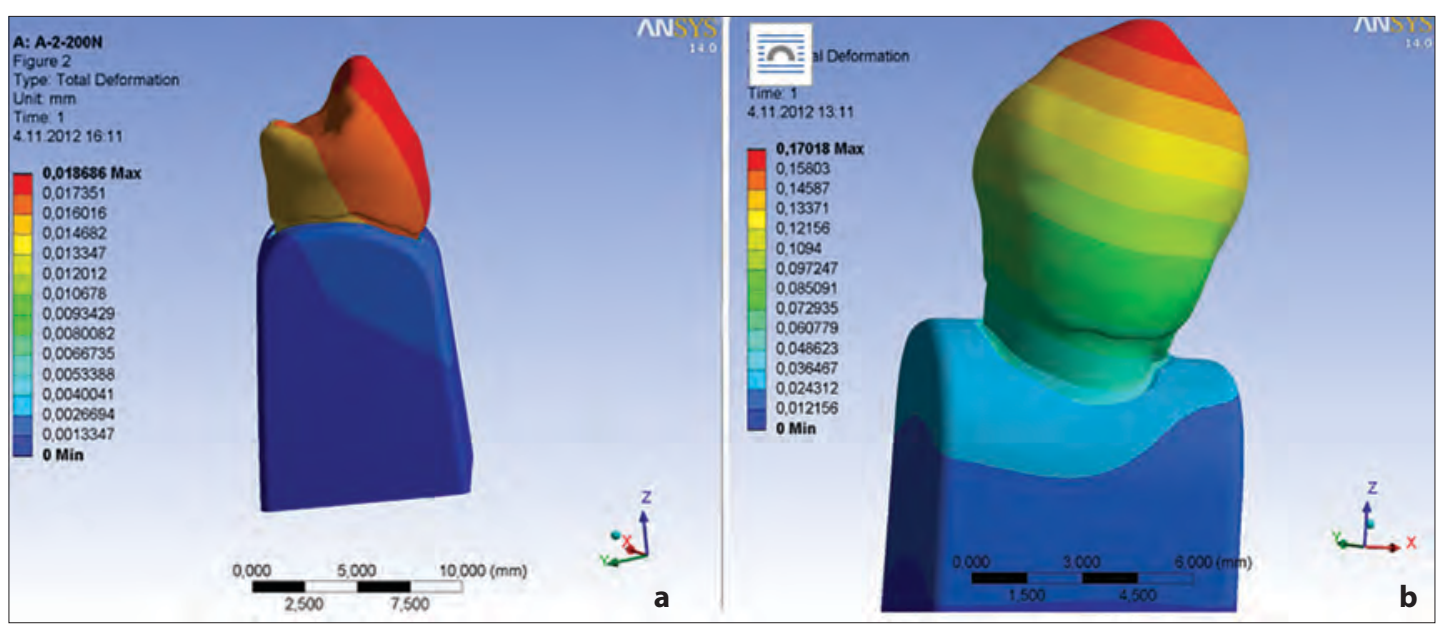

Figure 5. Distribution of deformation under axial (a) and para-axial loads (b) of $200 \mathrm{~N}$. 


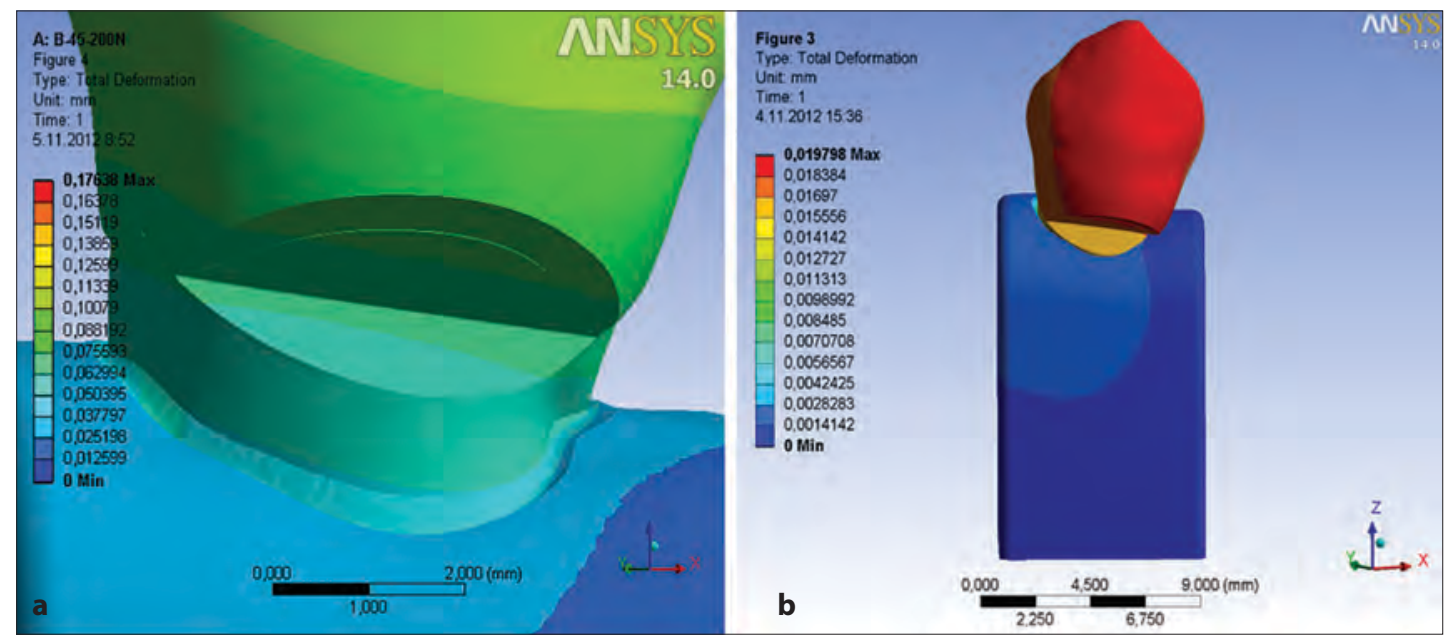

Figure 6. Type of deformation under a paraxial and axial load of $200 \mathrm{~N}$ on the tooth with a lesion. The highest values of deformation of the teeth were seen on the model with a cervical lesion, under an axial load at 0.019 $\mathrm{mm}$, paraxial load at $0.176 \mathrm{~mm}$.

compared to cases when an axial load occurs $(0.019 \mathrm{~mm})$ (Figure 6).

\section{Discussion}

The highest stress on the intact tooth model under both types of load was observed in the occlusal contact regions, under an axial load of $205 \mathrm{MPa}$, and a paraxial load of $220.02 \mathrm{MPa}$ (Figures 1 and $2 \mathrm{a}$ and $\mathrm{b}$ ). The activity of the occlusal load led to significant stress in the cervical region of the teeth. The von Mises stress measured in the cervical part of the tooth under axial load was up to $12 \mathrm{MPa}$, while in the same part of the tooth under paraxial load the stress measured was more than $50 \mathrm{MPa}$. Similar findings were presented by Rees (10), De las Casasa (11), Tanaka (12) and Kuroa (13).

It is known that the dental structures, especially the enamel, have significantly greater compressive strength than tensile, and are much more sensitive to tensile straining than compressive (14). Our findings showed that the buccal surface of the tooth is exposed to the more unfavorable type of stress - tensile stress. In the study by Rees (15), Yaman (16) and Borčić (17) the values of Maximum Principal Stress on the buccal side of the tooth range from 60-90 MPa, and are similar to our findings, where the Maximum Principal Stress noted on the buccal side of the tooth (tensile stress) was 94.998 $\mathrm{MPa}$ (Figure 4).

By analyzing the mechanisms of the formation of abfraction lesions, Rees and Hammadeh (18) developed a theory about the possible undermining of the enamel due to the concentration of force on the enameldentin border. Our analysis confirms the observations of these authors that the stress in the cervical part of the teeth is greater in the sub-surface than in the surface zone of the enamel, which is clearly visible on the images of the sagittal section of the tooth (Figure $3 b$ ).

If we compare models with a cervical lesion and the intact tooth model, we can see different types of stress distribution. The breakdown of the enamel and dentine continuity on the model with a lesion caused increased stress, with a concentration of stress around the apex/bottom of the lesion. The values of stress were higher in all dental tissues compared to the intact tooth, while the stress values under paraxial loading at the 
bottom of the lesion were very high, measuring 266.13 MPa (Figure 3a). The results of this analysis show that the bottom of the cervical lesions concentrates stress under loading, and further exposure of the lesion to stress will lead to the lesion's progression.

The type of deformation of the tooth model depended on the type of load. The deformation values of the tooth under paraxial loading were $\approx 10$ times greater than the deformation value of the tooth under axial load (Figures 5 and 6). The maximum values of tooth deformation were seen in the tooth model with a cervical lesion (Figure 6). The most prominent deformations were seen in the area of the tooth crown, especially on the occlusal surface and the tip of the buccal cusp. The deformation values decreased as they approached the apical area (Figure 5b).

Axial load caused the greatest deformation on the buccal surface of the tooth. The deformation decreased moving towards the lingual side (Figure 5a). This finding of tooth deformation, even in the central occlusion, indicates that the morphology, as well as the occlusion, of the mandibular first premolar is specific and often leads to an unfavorable distribution of stress, which could be the reason for the frequent finding of noncarious cervical lesions on these teeth. Various types of FEM models with NCCL of different depth and/or different shape could give more information on progression of NCCL.

\section{Conclusions}

The greatest influence on the intensity of stress in dental tissues comes from the type of load on the teeth. The values of the measured stress are greater under the action of a paraxial load. Occlusal loading leads to significant stress in the cervical part of the teeth, although this region is not directly exposed to the effect of mastication forces. The values of stress in an abfraction lesion measured under a paraxial load are extremely high. Lesions with expressed geometric discontinuity (V lesions) lead to the concentration of high stresses at the bottom of the tooth. Exposing the lesion to further stress will lead to its deepening. The total deformation of the entire tooth under paraxial load was $\approx 10$ times higher than the deformation of the tooth under axial load. The pattern of the deformation of the teeth differs depending on the type of load. The greatest deformation under load was measured on the tooth model with an existing abfraction lesion. Although FEM analysis gives realistic simulation and stress measurements, biological variations of tooth morphology, different types of intercuspidation and habitual occlusion are factors that limit this type of analysis. Also, the acting occlusal force in this research is assumed as static, while in reality there is always dynamic force, with a relatively large number of repetitions.

\section{What Is Already Known on this Topic:}

Noncarious cervical lesions (NCCL) are more often found on the mandibular first premolars compared to other types of teeth. FEM analysis has proved to be a useful method for experimental research on mechanical forces in human tissues.

What this Study Adds:

Numerous studies by means of FEM analysis have mainly focused on the biomechanics of bone tissue. This study contributes to understanding the connection between the specific morphology of the mandibular first premolar and the action of axial and paraxial forces on the development and progression of NNC lesions.

Authors' Contributions: Conception and design: AAV and SJ; Aqusition, analysis and interpretation of data: AAV, SJ, SZ; Drafting the article: AAV, SJ, SZ; Revising it criticaly for important intellectual content: AB, AGG and SŠ. Approved final version of the manuscript: AAV, SJ, SZ.

Acknowledgement: Authors would like to thank Prof. Alen Topčić and Prof. Edin Cerjaković from the University of Tuzla, for their help and scientific remarks during the production of the FEM analysis model.

Conflict of Interest: The authors declare that they have no conflict of interest. 


\section{References}

1. Vuković A, Zukić S, Bajsman A, Selmanagić A. Basics of teeth morphology and dental anthropology [in Bosnian]. 1st ed. Sarajevo: Faculty of Dentistry University of Sarajevo; 2013.

2. Ash MM. Wheeler's Dental anatomy, Physiology and Occlusion. 7th ed. Philadelphia: W.B. Saunders Company; 1993.

3. Jakupović S, Vuković A, Korać S, Tahmiščija I, Bajsman A. The prevalence, distribution and expression of noncarious cervical lesions (NCCL) in permanent dentition. Mat Soc Med. 2010;22(4):2004.

4. Aw C, Lepe X, Johnson GH, Mancl L. Caracteristics of noncarious cervical lesions, A clinical investigation. JADA. 2002;133(6):725-33.

5. Khan F, Young WG, Shahabi S, Daley TJ. Dental cervical lesions associated with occlusal erosion and attrition. Australian Dent J. 1999;44(3):17686.

6. Borčić J, Anić I, Urek MM, Ferreri S. The prevalence of non-carious cervical lesions in permanent dentition. J Oral Rehabil. 2004;31(2):117-23.

7. Grippo JO. Abfractions: a new classification of hard tissue lesions of teeth. J Esthet Dent. 1991 Jan-Feb;3(1):14-9.

8. Palamara J E A, Palamara D, Messer HH, Tyas M J. Tooth morphology and characteristics of noncarious cervical lesions. J Dent. 2006;34(3):185-94.

9. Jakupović S, Anić I, Ajanović M, Korać S, Konjhodžić A, Džanković A, et al. Biomechanics of cervical tooth region and noncarious cervical lesions of different morphology; three-dimensional finite element analysis. Eur J Dent 2016;10(3):413-8.
10. Rees JS. The effect of variation in occlusal loading on the development of abfraction lesions: a finite element study. J Oral Rehabil. 2002;29(2):188-93.

11. De Las Casas EB, Cornacchia TP, Gouvêa PH, Cimini CA Jr. Abfraction and anisotropy--effects of prism orientation on stress distribution. Comput Methods Biomech Biomed Engin. 2003 Feb;6(1):65-73.

12. Tanaka M, Naito T, Yokota M, Kohno M. Finite element analysis of the possible mechanism of cervical lesion formation by occlusal force. J Oral Rehabil. 2003;30(1):60-7.

13. Kuroe T, Itoh H, Caputo A A, Konuma M. Biomechanics of cervical tooth structure lesions and their restoration. Quint Int. 2000;31(4):267-74.

14. Geramy A, Sherafoddin F. Abfraction: 3d analysis by means of the finite element method. Quint Int. 2003;34(7):526-33.

15. Rees J S, Hammadeh M, Jagger D C. Abfraction lesion formation in maxillary incisors, canines and premolars: A finite element study. Eur J Oral Sci. 2003;111(2):149-54.

16. Yaman S. D, Sahin M, Aydin C. Finite element analysis of strength characteristics of variou resin base restorative materials in Class $\mathrm{V}$ cavities. J Oral Rehabil. 2003;30(6):630-41.

17. Borčić J, Anić I, Smojver I, Ćatić A, Miletić I, Ribarić S.P. 3D finite element model and cervical lesion formation in normal occlusion and in malocclusion. J Oral Rehabil. 2005;32(7):504-10.

18. Rees JS, Hammadeh M. Undermining of enamel as a mechanisam of abfraction lesion formation: a finite element study. Eur J Oral Sci. 2004;112(4): 347-52. 